\title{
A ENFERMEIRA PEDIATRA CUIDANDO DE CRIANÇAS/ ADOLESCENTES COM SÍNDROME ALCOÓLICA FETAL (SAF)
}

\author{
The Pediatrician Nurse Taking Care of Children/ \\ Adolescents with Fetal Alcoholic Syndrome (SAF) \\ La Enfermera Pediatra Cuidando de Niños/ \\ Adolescentes con el Síndrome Alcohólico Fetal (SAF)
}

Flávia Atanazio do Nascimento ${ }^{1}$

José Mauro Braz de Lima ${ }^{4}$

\author{
Mariana Coutinho de Almeida² \\ Rosângela da Silva Santos ${ }^{5}$
}

Jurema Gouvea de Souza ${ }^{3}$

\section{Resumo}

Estudo realizado em uma instituição pública de Neurologia com seis crianças que apresentavam diagnóstico de síndrome alcoólica fetal (SAF). Os Objetivos foram: Descrever as necessidades humanas básicas afetadas em crianças com SAF; identificar as áreas de desenvolvimento defasadas; e avaliar o desenvolvimento das crianças. Metodologia: Pesquisa qualitativa, aprovada pelo Comitê de Ética e Pesquisa do Hospital Escola São Francisco de Assis (HESFA) e Escola de Enfermagem Anna Nery. 0 desenvolvimento infantil foi avaliado com a Escala de Desenvolvimento de Heloisa Marinho. Resultados: Todas as crianças apresentaram defasagem na área mental, social e física. As crianças apresentaram alheamento, timidez, recusa em verbalizar e manter contato visual, hiperatividade; dificuldade de aprendizagem; dificuldade na coordenação motora; timidez; e agitação psicomotora. As necessidades afetadas foram: hidratação, higiene oral, higiene corporal, comunicação, coordenação motora, aprendizagem, educação alimentar e visão. Todos apresentaram grande defasagem em relação à idade cronológica, idade de desenvolvimento e idade gráfica e baixo quociente de desenvolvimento.

Palavras-chave: Enfermagem. Desenvolvimento Infantil. Síndrome Alcoólica Fetal.

\section{Abstract}

Study accomplished in a public Institution of Neurology, with six children with Fetal Alcoholic Syndrome (SAF). The Objectives were: To describe the basic human needs affected in children with Fetal Alcoholic Syndrome (SAF). To identify the areas of development harmed. To evaluate the children's development with Fetal Alcoholic Syndrome (SAF). Methodology: Researches qualitative. The study was approved by the Committee of Ethics and Research of Hospital Escola São Francisco de Assis (HESFA) and school of Nurse Anna Nery. The development was evaluated by the scale of development of Heloisa Marinho. Results: All of the children presented discrepancy in the Mental; Social and Physical area. Children presented alienation, shyness, and refuse in to verbalize and to maintain visual contact and hyperactivity; learning difficulty; difficulty in the motive coordination; shyness; motive agitation. The affected needs were: hydration, oral hygiene, corporal hygiene, communication, motive coordination, learning, alimentary education, vision. All presented great discrepancy in relation to the chronological age, development age, graphic age and low development quotient.

Keywords:

Nursing. Infantile development. Fetal Alcoholic Syndrome.

\section{Resumen}

El estudio fue realizado en una Institución pública de Neurología, con seis niños con el Síndrome Alcohólico Fetal (SAF). Los Objetivos eran: Describir las necesidades humanas básicas afectadas en los niños con el Síndrome Alcohólico Fetal (SAF). Identificar las áreas de desarrollo dañadas. Evaluar el desarrollo de los niños con el Síndrome Alcohólico Fetal (SAF). Metodología: Investigación Cualitativa, aprobada por el Comité de Ética y Investigación del Hospital Escuela São Francisco de Assis (HESFA) y Escuela de Enfermería Anna Nery. El desarrollo infantil se evaluó por la Escala de Desarrollo de Heloisa Marinho. Resultados: Todos los niños presentaron defasaje en el área mental, social y física. Los niños presentaron alienación, timidez, y negación en verbalizar y mantener contacto visual, hiperactividad; dificultad de aprendizaje; dificultad en la coordinación motora; timidez y agitación psicomotora. Las necesidades afectadas fueron: hidratación, higiene oral, higiene corpórea, comunicación, coordinación motora, aprendizaje, educación alimentar, visión. Todos presentaron gran defasaje cuanto a la edad cronológica, edad de desarrollo y edad gráfica y cociente de desarrollo bajo.

\footnotetext{
$\overline{{ }^{1} \text { Acadêmica de Enfermagem }}$ do $7^{\circ}$. Período do Curso de Graduação em Enfermagem e Obstetrícia da Escola de Enfermagem Anna Nery/UFRJ. Voluntária na pesquisa; ${ }^{2}$ Acadêmica de Enfermagem do $7^{\circ}$. Período do Curso de Graduação em Enfermagem e Obstetrícia da Escola de Enfermagem Anna Nery/UFRJ. Membro do Núcleo de Pesquisa em Saúde da criança. NUPESC/DEMI/EEAN/UFRJ. Bolsista de IC PIBIC/UFRJ; ${ }^{3}$ Professora Assistente do DEMI/EEAN/UFRJ. Doutora em Enfermagem; ${ }^{4}$ Médico Neurologista. Coordenador do Centro de Estudos dos Problemas Relacionados ao Uso Abuso de Álcool (CEPRAL). PHd; ${ }^{5}$ Professora Titular do DEMI da Escola de Enfermagem Anna Nery/UFRJ. Pesquisadora I C do CNPq/FAPERJ/Núcleo de Pesquisa em Saúde da Criança e adolescente (NUPESC)/Núcleo de Pesquisa em Saúde da Mulher (NUPESM). E-mail: roiva@superig.com.br
} 


\section{CONSIDERAÇÕES INICIAIS}

0 interesse em realizar o presente estudo teve origem na experiência prática de duas enfermeiras obstetras e um médico neurologista ao assistir, em uma instituição pública na Cidade do Rio de Janeiro, mulheres que apresentavam consumo abusivo de bebida alcoólica e tiveram filhos com a síndrome alcoólica fetal (SAF). Propomos como objeto de estudo as necessidades humanas básicas afetadas em crianças com SAF.

No Brasil, as primeiras referências à SAF foram feitas em meados da década de 1980, despertando a atenção para a importância e gravidade do problema 1 . A importância da SAF decorre do fato de ela causar danos irreversíveis ao cérebro, porém, ser totalmente prevenível se a gestante se abstiver do uso de bebida alcoólica durante o período gestacional. Atualmente, ainda não é conhecido um nível seguro de consumo de bebida alcoólica durante a gravidez; por esta razão, gestantes devem ser alertadas para a completa abstenção do álcool.

0 mundo do feto enquanto ele está no útero materno é um ambiente confortável e protegido, porém não imune às influências do ambiente externo. A síndrome alcoólica fetal foi observada e descrita, primeiramente, na França ${ }^{2}$ e, posteriormente, ratificada em trabalhos científicos nos EUA ${ }^{3}$. É caracterizada clinicamente em sua forma clássica por três grupos de sintomas: atraso do desenvolvimento pré e/ou pósnatal (baixo peso, baixa estatura ou circunferência craniana menor que o percentual 10 para a idade gestacional); comprometimento do sistema nervoso central (SNC), defeitos neurológicos e retardo mental de grau variável - atraso no desenvolvimento intelectual, principalmente distúrbios da aprendizagem e do comportamento; déficit da memória e da atenção; hiperatividade; impulsividade e agressividade; dismorfias craniofaciais: microcefalia (circunferência craniana pequena), microftalmia, micrognatia (área maxilar achatada) e lábio superior fino.

Atualmente o uso de bebida alcoólica por mulheres é muito comum, e, como conseqüência, é elevado o número das que bebem durante a gestação. Observamos em nosso cotidiano assistencial que as gestantes durante a realização do prénatal referem uso abusivo de bebida alcoólica sem que tenham a noção de que estão ingerindo quantidade capaz de trazer malefícios para o bebê. Já adultos jovens saudáveis que utilizam moderadamente bebida alcoólica podem se beneficiar em relação ao bem-estar, à boa saúde e até mesmo na prevenção de várias doenças ${ }^{4}$. É difíicil definir, no entanto, o uso moderado de bebidas alcoólicas. 0 senso comum estabelece que beber moderadamente é beber socialmente. Mas o próprio conceito de beber socialmente é subjetivo e depende do ponto de vista de cada pessoa, e significa beber o aceitável pela sociedade. Por outro lado, beber com moderação trás implícita a idéia de que não há malefício para quem consome, o que não é correto. Uma dose de bebida alcoólica contém de 8 a 13 gramas de etanol. Para homens, a OMS preconiza que o consumo aceitável deve ser de até 15 doses/semana e, para as mulheres, 10 doses/ semana. Uma dose equivale a aproximadamente $285 \mathrm{ml}$ de cerveja, $120 \mathrm{ml}$ de vinho e $30 \mathrm{ml}$ de destilado (uísque, vodca, pinga) ${ }^{4}$.
Cabe ressaltar que o consumo moderado varia de pessoa para pessoa, depende diretamente do contexto, da experiência, da tolerância, do metabolismo, da vulnerabilidade genética, do estilo de vida, do tempo de consumo da bebida, ou seja, da quantidade de bebida que o indivíduo suporta beber sem se intoxicar.

Gestantes e lactantes que consomem bebida alcoólica, mesmo em forma moderada, durante a gestação, expõem-se a duas doenças sérias: a síndrome alcoólica fetal e o efeito fetal do álcool. Quando a gestante ingere bebida alcoólica em aproximadamente 40 a 60 minutos, o mesmo teor de álcool do organismo materno é encontrado no sangue fetal. Deste modo, a mãe ingere por via oral a bebida alcoólica e o feto recebe por via endovenosa (via placentária) o mesmo teor alcoólico, ocasionando intoxicação no sangue fetal.

0 efeito de bebidas alcoólicas em crianças e jovens que ainda não atingiram a maturidade física completa pode acarretar "obstrução do crescimento emocional e psicológico e contribuir para uma variedade de doenças do sistema nervoso central, podendo também prejudicar a função vital de órgãos internos." ${ }^{4}$ No entanto, em certos casos, o próprio médico obstetra recomenda 0 uso de bebida alcoólica de forma moderada em reuniões sociais e os meios de divulgação exibem reportagens apresentando casos em que o uso freqüente $\mathrm{e}$ moderado do vinho pode ser responsável por uma vida longa e saudável. "A maior dificuldade em conscientizar as pessoas dos danos que ele pode causar está no fato de que a mídia enfatiza, apenas, seu lado positivo: bebidas alcoólicas são apresentadas como boas e relacionadas às situações de felicidade, alegria, aceitação social, etc" 5 .

Desde modo, a mídia enfatiza os aspectos negativos das drogas ilícitas e os positivos das lícitas. Para a autora, existe uma tendência a "tratar as drogas de maneira emocional, ao invés de tratá-la de modo racional e científico, ajudando, assim, a criar uma representação social inapropriada das substâncias psicoativas encontrada no mundo todo. E isto é o que faz as pessoas acreditarem que 0 álcool não causa dano algum"5.

A partir do exposto e da experiência prática de duas enfermeiras e um médico neurologista, preocupados com a premissa de que o consumo de bebida alcoólica durante a gestação, mesmo de forma moderada, pode comprometer 0 desenvolvimento fetal e infantil, questionamos: Que alterações do desenvolvimento infantil são apresentadas por crianças com síndrome alcoólica fetal (SAF)? Que necessidades humanas básicas estão afetadas na criança com SAF? A partir desses questionamentos, foram elaborados os seguintes objetivos: Descrever as necessidades humanas básicas afetadas em crianças com SAF; identificar as áreas de desenvolvimento defasadas; e avaliar o desenvolvimento de crianças com SAF. Nos últimos dez anos, a atenção médica foi despertada por outro importante e grave problema, até então pouco suspeitado, que são as repercussões fetais teratogênicas do alcoolismo. 0 uso de álcool durante a gravidez, algumas vezes nos primeiros meses, quando a mulher ainda nem sabe que está grávida, tem sido apontado como responsável por uma série de defeitos congênitos, de maior ou menor gravidade, responsável por 
atraso no desenvolvimento psicomotor (prematuridade), além de complicações neurológicas, às vezes manifestadas por distúrbios cognitivos e/ou comportamental. De um modo geral, as repercussões deletérias da bebida alcoólica sobre o feto têm sido objeto de estudo em alguns países desenvolvidos onde tem suscitado grande interesse face às repercussões nas áreas da saúde, educação e economia.

Apesar de ser um problema reconhecido ao longo de muitos anos, observamos que ainda é incipiente a atenção dispensada ao aprofundamento das questões apresentadas, no sentido de combater os efeitos maléficos causados pelo consumo do álcool durante a gravidez; diminuir o número de recém-nascidos atingidos; e esclarecer a população em idade fértil dos riscos a que estão expostas ao ingerir bebida alcoólica durante a gestação. É reduzido o número de publicações, principalmente, na área da Enfermagem. Portanto, um maior conhecimento do assunto é necessário para que se possam estabelecer políticas e estratégias de prevenção efetiva.

Um grande número de crianças apresenta distúrbios no desenvolvimento e no comportamento devidos ao consumo prejudicial de bebida alcoólica por suas mães durante a gravidez. No Brasil, não se têm estatísticas confiáveis em relação a estes dados porque o diagnóstico de SAF é difícil. Muitos pediatras não estão alertas para o problema, e o diagnóstico é confirmado após eliminar todas as outras possíveis causas para as malformações faciais, a microcefalia e o atraso no desenvolvimento psicomotor. Deste modo, o diagnóstico da SAF ocorre por exclusão dos demais diagnósticos e, via de regra, se dá no período escolar, ocasião em que as seqüelas são evidentes em virtude das defasagens no desenvolvimento infantil em função da não-implementação de uma intervenção precoce.

Considerando-se que a Política de Saúde atual tem como prioridade a redução das taxas de mortalidade materna, fetal e infantil e a prevenção dos agravos resultantes da violência sexual contra mulheres e adolescentes e com os direitos sexuais e reprodutivos ${ }^{6}$, este estudo oferece subsídios para a prática assistencial, instrumentalizando profissionais da área de saúde para atuar com crianças que apresentam SAF.

Contribui, também, com a academia no sentido de retroalimentar os conteúdos programáticos de disciplinas dos cursos de graduação e pós-graduação (latu e stricto sensu) na área de saúde da criança.

\section{METODOLOGIA}

0 presente estudo utilizou a abordagem qualitativa que permite trabalhar com "o universo de significados, motivos, aspirações, crenças, valores e atitudes, o que corresponde a um espaço mais profundo das relações, dos processos e dos fenômenos, que não podem ser reduzidos à operacionalização de variáveis."

A opção pela abordagem qualitativa deve-se ao fato de estarmos interessados em conhecer as necessidades humanas básicas afetadas em crianças com SAF e identificar as áreas de desenvolvimento defasadas.

0 cenário de pesquisa foi o Centro de Ensino, Pesquisa e Referência de Alcoologia e Adictologia (CEPRAL) de um hospital de Neurologia no Rio de Janeiro. Este Centro foi criado para desenvolver pesquisas e atendimento de pessoas com as conseqüências da exposição ao álcool durante a gravidez, apresentadas por crianças em idade pré-escolar, escolar e adolescente, sendo, também, campo de estágio para capacitação de profissionais e de desenvolvimento de pesquisa em nível de pós-graduação.

0 Núcleo de Atenção à Síndrome Alcoólica Fetal - NASAF apresenta um programa de atendimento ambulatorial que conta com equipe multidisciplinar que atende: a criança e 0 adolescente que apresentam características neuropsicológicas e comportamentais que sinalizam a hipótese de diagnóstico de SAF; as gestantes do ambulatório de pré-natal e os recémnascidos; e família, no sentido do diagnóstico e orientação terapêutica, além de encaminhar as mães alcoolistas e/ou usuárias de outras drogas para o ambulatório de tratamento dos problemas relacionados ao uso abusivo de dependência de álcool (CEPRAL) e/ou a outros locais de referência.

Os sujeitos do estudo foram seis crianças/adolescentes com SAF em atendimento no Núcleo de Atenção à Síndrome Alcoólica Fetal - NASAF de uma instituição pública de Neurologia no Rio de Janeiro. Cabe registrar que os sujeitos encontravam-se na faixa etária de 12,10 anos (doze anos e dez meses) e 17,3 anos (dezessete anos e três meses). Cronologicamente, tratavase de pré-adolescentes e adolescentes, porém, após a realização da Consulta de Enfermagem, detectamos defasagem no desenvolvimento das mesmas, fato este que nos levou a adotar a Escala de Desenvolvimento de Heloisa Marinho, e detectamos que as mesmas apresentavam Idade de Desenvolvimento entre 2,11 anos (2 anos e 11 meses) e 8,4 anos (8 anos e 4 meses).

Os critérios de inclusão no estudo foram: ter diagnóstico de síndrome alcoólica fetal (SAF); estar inscrita no cenário de pesquisa; comparecer às consultas agendas. Não foi utilizado critério de exclusão. As seis crianças/adolescentes com SAF que compareceram para atendimento no período de março a julho de 2006, no Instituto de Neurologia, e que aceitaram participar da pesquisa foram sujeito do estudo. Cabe registrar que a adolescente Cristina participou de três encontros, ocasião em que a consulta de enfermagem foi realizada. No dia aprazado para aplicação da Escala de Desenvolvimento, a mesma estava na casa de seus familiares. Por esta razão, os dados constantes no estudo foram os obtidos no exame físico durante a realização da consulta de enfermagem. Todas as crianças aceitaram e se integraram às atividades imediatamente, no entanto, em função do nível de compreensão, as mesmas não assinaram o Termo de Consentimento livre e Esclarecido. Solicitamos autorização às mães e/ou profissionais dos abrigos em que algumas eram criadas.

As mães e/ou responsáveis pelas crianças autorizaram a participação das mesmas na pesquisa através de carta explicativa acerca dos objetivos e importância do estudo e termo de consentimento livre esclarecido, garantindo o sigilo das informações obtidas e preservação da identidade, conforme a resolução n 196/96 do Conselho Nacional de Saúde que dispõe sobre as diretrizes e normas regulamentadoras de pesquisa envolvendo seres humanos. À criança/adolescente foi 
solicitado que escolhesse um codinome para constar no estudo, e desta forma garantiu-se o anonimato preconizado na Resolução. Uma cópia do Projeto de Pesquisa foi encaminhada ao Comitê de Ética e Pesquisa do Hospital Escola São Francisco de Assis - HESFA e Escola de Enfermagem Anna Nery, e o projeto foi aprovado em 11 de maio de 2005, Protocolo nº. 020/05.

A coleta de dados ocorreu durante a realização da consulta de enfermagem, aprazada mensalmente, às quintas feiras no horário de 14:00 às 17:00. Para esta coleta, utilizamos como instrumento o formulário de exame físico adotado no Programa Curricular I "A Criança, a Escola e Eu" da Escola de Enfermagem Anna Nery/UFRJ e a Escala de Desenvolvimento Infantil de Heloisa Marinho. As crianças/adolescentes foram avaliadas por duas acadêmicas do Curso de Graduação em Enfermagem da EEAN/UFRJ, supervisionadas por docente de Enfermagem, as quais realizaram anamnese e exame físico, com o intuito de identificar as necessidades humanas básicas afetadas e os problemas de saúde e de enfermagem, e aplicaram a Escala de Desenvolvimento de Heloisa Marinho às crianças com SAF.

Após a realização da consulta de enfermagem, foi aplicada a Escala de Desenvolvimento de Heloísa Marinho para avaliar o desenvolvimento infantil e detectar possíveis defasagens no desenvolvimento. A Escala de Desenvolvimento de Heloísa Marinho foi criada em 1957 a partir de aulas de desenvolvimento infantil na Associação de Pais e Alunos de Excepcionais - APAE. Seus 15 anos de experiência utilizando as escalas de Gesell, Bühler e Binet-Simon forneceram-lhe embasamento para que organizasse uma seqüência de provas que propiciasse identificar o nível do desenvolvimento infantil a partir da observação do comportamento livre da criança nos primeiros meses e anos de vida. Durante 36 anos, "estudantes de nível superior colheram os dados e computaram a estatística no Instituto Técnico do Colégio Bennett e no Curso de Pedagogia Especial do Instituto de Educação do Rio de Janeiro ${ }^{7 " .}$.

A Escala de Desenvolvimento de Heloísa Marinho avalia o desenvolvimento da criança de 0 (zero) a 8,11 anos (oito anos e onze meses) em três grandes áreas do desenvolvimento: Físico, Mental e Social7.

A partir dos dados obtidos, foi elaborado um plano de intervenção compatível com o desenvolvimento infantil apresentado pelas crianças com SAF.

No período de março a julho de 2006, foram realizados três encontros com cada criança/adolescente com SAF na Instituição de Neurologia. Durante este período, realizamos o exame físico e aplicamos a Escala de Avaliação do Crescimento e Desenvolvimento de Heloisa Marinho. Os dados obtidos foram provenientes da folha de anamnese dos prontuários das crianças avaliadas; da observação e exame físico; dos desenhos; das manifestações verbais das mesmas durante os encontros na consulta de enfermagem, bem como, informações fornecidas pelas mães e profissionais dos abrigos em que algumas crianças/ adolescentes eram criadas.

A realização do exame físico das crianças permitiu a elaboração do diagnóstico de enfermagem em relação às necessidades apresentadas pelas crianças. A aplicação da Escala de Avaliação do Desenvolvimento Infantil de Heloisa
Marinho possibilitou comparar a idade cronológica com a idade de desenvolvimento e o quociente de desenvolvimento da criança, detectando a (s) área (s) que se encontrava (m) defasada(s), e elaborar um plano de intervenção com o objetivo de estimular as mesmas.

Todos os dados coletados foram identificados, agrupados, codificados, decodificados e analisados por unidades temáticas. "A análise temática consiste em descobrir os núcleos de sentido que compõem uma comunicação cuja presença ou freqüência signifiquem alguma coisa para o objetivo analítico visado".

Deste modo, três unidades temáticas foram identificadas: o desenvolvimento das crianças/adolescentes avaliados com a Escala de Desenvolvimento de Heloisa Marinho; características do desenvolvimento das crianças/adolescentes com SAF em relação à comunicação; enfermeira pediatra, a criança com SAF e a sistematização da assistência de enfermagem.

\section{0 desenvolvimento das Crianças/Adolescentes Avaliados com a Escala de Desenvolvimento de Heloisa Marinho:}

A seguir apresentamos a caracterização do grupo de crianças examinadas e que eram atendidas na Instituição de Neurologia.

\section{Tabela 1:}

Caracterização das Crianças/Adolescentes examinados.

\begin{tabular}{cccccc}
\hline Identificação & I.C. & I.D. & Q.D. & I.G. & $\begin{array}{c}\text { Áreas } \\
\text { defasadas }\end{array}$ \\
\hline Cristina & - & - & - & - & - \\
Joana & 13,5 & 7,6 & 50,7 & 5,9 & $\mathrm{~F} / \mathrm{M} / \mathrm{S}$ \\
Carmem & 17,3 & 8,4 & 55,88 & 9,6 & $\mathrm{M} / \mathrm{S}$ \\
Antônio & 16,11 & 2,11 & 18,23 & - & $\mathrm{F} / \mathrm{M} / \mathrm{S}$ \\
Eliel & 15,6 & 3,11 & 24,44 & - & $\mathrm{F} / \mathrm{M} / \mathrm{S}$ \\
Wanderley & 12,10 & 7,8 & 63,88 & 5,3 & $\mathrm{M}$ \\
\hline
\end{tabular}

Legenda: $\mathrm{F}=$ Física; $\mathrm{M}=$ Mental; $\mathrm{S}=$ Social. I.C= Idade Cronológica; I.D=Idade de Desenvolvimento; I.G = Idade Gráfica

Fonte: Dados obtidos com a aplicação da Escala de Avaliação do Desenvolvimento Infantil de Heloisa Marinho.

A Tabela 1 evidencia que a maioria dos sujeitos do estudo atendida no referido serviço cronologicamente é adolescente. Encontra-se na faixa etária de 13,5 anos (treze anos e cinco meses) a 17,3 anos (dezessete anos e três meses). Observase que apenas uma delas ainda é considerada criança (segundo o Estatuto da Criança e do Adolescente $\left(E C A^{9}\right)$, criança é "a pessoa até doze anos de idade incompletos, e adolescente é aquela entre doze e dezoito anos de idade" $)^{9}$. A idade de desenvolvimento variou de 2,11 anos (dois anos e onze meses) a 8,4 anos (oito anos e quatro meses). A idade gráfica variou de 5,3 anos (cinco anos e três meses) a 9,6 anos (nove anos e seis meses). É importante registrar que em dois adolescentes não foi possível avaliar a idade gráfica (encontrava-se em fase de rabiscação). 0 quociente de desenvolvimento ficou entre 18,23 e 63,88. Ressalta-se que a faixa do quociente de desenvolvimento considerada "normal" encontra-se entre 90 e 1207, portanto, os sujeitos do estudo encontram-se bem abaixo da faixa de normalidade.

Em relação ao gênero, observa-se que 3 eram do sexo feminino e 3 , do sexo masculino. Após a aplicação da Escala 
de Desenvolvimento de Heloisa Marinho evidenciou-se que todas apresentavam defasagem na área mental, social e física.

Tabela 2:

Características do Desenvolvimento das Crianças/Adolescentes com SAF em relação à Comunicação.

\begin{tabular}{cc}
\hline Identificação & Comunicação \\
\hline Cristina & Introversão/Acentuada Timidez/alheamento \\
Joana & Introversão/Acentuada Timidez/alheamento \\
Carmem & Introversão/ Timidez \\
Antônio & Introversão/Acentuada Timidez/alheamento \\
Eliel & Introversão/Acentuada Timidez/alheamento \\
Wanderley & Hiperatividade \\
\hline
\end{tabular}

Fonte: Dados obtidos na sistematização da assistência de enfermagem após a realização do exame físico durante consulta de enfermagem.

Segundo a Tabela 2, o padrão de comportamento das crianças variou de acordo com o lugar em que se encontravam. Quando avaliadas na Instituição de Neurologia apresentaramse tímidas (algumas em excesso), não olhavam no rosto das examinadoras. Ficavam sempre cabisbaixas, e não falavam com ninguém. Se alguém Ihe fazia uma pergunta não respondiam, continuavam olhando na direção do chão, em algumas ocasiões sorriam, mas não emitiam som. Procuravam, via de regra, o olhar do atendente do abrigo, da mãe ou responsável durante a realização da consulta de enfermagem ou em alguma atividade informal com as examinadoras.

É importante registrar que todas as crianças, quando avaliadas na Instituição de Neurologia, apresentaram padrão de comportamento voltado para si mesmas, evidenciando alheamento do contexto externo, timidez, recusa em verbalizar, recusa em manter contato visual, etc. Quando avaliadas no abrigo em que residiam, interagiram com as examinadoras, conversaram com as atendentes do abrigo, brincavam e conversavam entre si e com as examinadoras.

0 alheamento ocorre quando a criança se esquiva, ou mesmo se recusa terminantemente a manter contato com outras pessoas, ou com qualquer outro aspecto do ambiente sócio-cultural no qual está inserida. Em sua manifestação mais leve, encontram-se crianças que não iniciam contato verbal, não respondem quando solicitadas, não brincam com outras crianças, ou mesmo mostram falta de interesse pelos estímulos ou acontecimentos do ambiente. É importante registrar que, embora não tenhamos observado, há informação dos responsáveis por algumas crianças de comportamentos agressivos.

Uma única criança foi identificada pela mãe como hiperativa, muito embora, ao exame físico e na interação durante a aplicação da Escala de Heloisa Marinho, tenha apresentado comportamento considerado "normal". Sabe-se que a criança hiperativa apresenta fundamentalmente uma inabilidade para controlar seu comportamento motor de acordo com as exigências nas diversas situações. Assim, apresenta uma constante mobilidade e agitação motora, o que também se torna grande empecilho para seu envolvimento com uma determinada ação ou tarefa.

0 álcool seria uma das principais causas de déficit de atenção e distúrbio de conduta (ansiedade, resistência para absorver regras sociais, compulsividade, irritabilidade, maior dependência). As conseqüências são graves. 0 baixo rendimento escolar, por exemplo, causando repetência e exclusão da escola, é um dos fatores favoráveis para o surgimento de comportamento anti-social, o que acarretaria a prevalência de alguns fatores secundários provenientes da SAF durante a vida das pessoas, como comportamento sexual inapropriado; problemas com a lei; problemas relacionados ao uso e abuso do álcool e outras drogas; problemas com emprego, dentre outros ${ }^{10}$.

\section{Enfermeira Pediatra, a Criança com Síndrome Alcoólica Fetal (SAF) e a Sistematização da Assistência de Enfermagem}

Tabela 3:

Necessidades Humanas Básicas Afetadas nas Crianças/ Adolescentes com SAF.

\begin{tabular}{|c|c|c|c|c|c|c|c|}
\hline & $\begin{array}{c}\text { Crianças/ } \\
\text { Adolescentes }\end{array}$ & Cristina & Joana & Carmem & Antônio & & el Wanderley \\
\hline \multirow{4}{*}{ 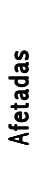 } & Hidratação & $x$ & $x$ & $x$ & $x$ & $x$ & \\
\hline & Higiene Oral & $x$ & & $x$ & $x$ & $x$ & $x$ \\
\hline & Higiene Corporal & $x$ & & $x$ & $x$ & $x$ & $x$ \\
\hline & Comunicação & $x$ & $x$ & $x$ & $x$ & $x$ & $x$ \\
\hline \multirow{4}{*}{ 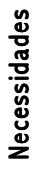 } & Coordenação motora & $x$ & $x$ & $x$ & $x$ & $x$ & $x$ \\
\hline & Aprendizagem & $x$ & $x$ & $x$ & $x$ & $x$ & $x$ \\
\hline & Educação alimentar & & $x$ & & & & \\
\hline & Visão & & & $x$ & $x$ & & \\
\hline
\end{tabular}

Fonte: Dados obtidos na sistematização da assistência de enfermagem após a realização do exame físico durante consulta de enfermagem.

A Tabela 3 evidencia que na criança/adolescente com SAF muitas são as necessidades humanas afetadas. 0 estudo mostrou que as necessidades afetadas na maioria dos sujeitos do estudo foram: hidratação; higiene oral; higiene corporal; comunicação; coordenação motora e aprendizagem. Observa-se que dessas seis necessidades, a coordenação motora e a aprendizagem estavam afetadas em todas as crianças/adolescentes. Já a visão estava afetada em dois adolescentes, e a educação alimentar, em apenas uma. Em relação ao grau de dependência, todas as crianças/adolescentes apresentaram dependência total ${ }^{11}$.

Os principais problemas de enfermagem identificados foram: dificuldade de aprendizagem; déficit no autocuidado banho/ higiene; baixa auto-estima; excesso de peso; dentes desalinhados; acentuada timidez; comunicação verbal prejudicada; desorientação nas esferas de tempo, espaço e pessoa; deficiente ingesta de líquidos; coordenação motora prejudicada; risco de trauma relacionado com dificuldades cognitivas e emocionais; comportamentos impróprios ou exagerados.

Considerando-se a individualidade e singularidade de cada criança/adolescente, os principais planos assistenciais foram: desenvolver análise de tarefa para a realização de Atividades da Vida Diária (AVD), como: higiene corporal adequada e higiene íntima durante o período menstrual; demonstrar e ensinar o processo de escovação dentária e hidratação oral; encaminhar ao serviço odontológico do Hospital Universitário e estimular o comparecimento ao serviço odontológico para correção da arcada dentária; promover atividade recreativa para orientar quanto à ingestão de alimentos com pouca gordura e à ingesta 
de legumes, verduras e frutas e desestimular a ingesta de doces e massas; promover brincadeiras e atividades que estimulem a relação interpessoal; estimular a realização de exercícios escolares para inseri-lo no grupo social da escola; encaminhar ao atendimento com fonoaudióloga e psicológico; propor para o cliente, durante as atividades recreativas, a prática de brincadeiras como amarelinha e pular corda; orientar a execução de pinturas e modelagem; estimular as potencialidades da criança/adolescente; enfatizar comportamentos de adaptação positivos; incentivar a necessidade da aprendizagem.

Apesar do grande número de defasagens apresentadas por estas crianças/adolescentes, estabelecemos no plano assistencial prognósticos como metas a serem alcançadas.

Os principais prognósticos estabelecidos foram: a criança/ adolescente manterá o balanço hídrico equilibrado; apresentará pele hidratada; demonstrará autocuidado independente no banho/higiene; adotará uma escovação dentária apropriada e quantas vezes forem necessárias durante 0 dia; reduzirá 0 consumo de doces e massas; aumentará sua comunicação com outras pessoas; desenvolverá sua fala; desenvolverá habilidades manuais e coordenação motora; participará de atividades apropriadas para a idade dentro dos limites de sua capacidade; aumentará a interação com colegas e a família; freqüentará a escola com regularidade; diminuirá o isolamento social; demonstrará maior interesse em aprender.

As evoluções do Processo de Enfermagem foram omitidas por não ser possível realizar e avaliar as modificações decorridas após a implementação do plano de cuidados.

\section{Referências}

1. Lima JMB. Álcool e gravidez. Síndrome Alcoólica Fetal. Arq Bras Méd 1985; 59(1): 1 -2.

2. Lemoine P, Harousseau H, Borteyru JP, Meneut JC. Les enfants de parents alcooliques: Anomalies observees. A propos de 127 cas. Ouest Med 1968; 21:476-82.

3. Jones KL, Smith DW. Recognition of the fetal alcohol syndrome in early infancy. Lancet 1973; 2 (836): 999-1001.

4. Ministério da Saúde (BR). Álcool e redução de danos: uma abordagem inovadora para países em transição. Brasília (DF); 2004.

5. Ministério da Saúde (BR). 0 papel da mídia na promoção do uso responsável de álcool. Álcool e redução de danos: uma abordagem inovadora para países em transição. Brasília (DF); 2004.

\section{CONSIDERAÇÕES FINAIS}

0 estudo evidenciou que todas as crianças analisadas, apesar de não obterem uma idade gráfica e de desenvolvimento de acordo com sua idade cronológica, apresentavam um familiar ou grupo que favoreciam a sua aprendizagem.

A maioria destas crianças mora em abrigo, freqüenta escola de crianças normais, participa em turma de alfabetização de adultos. 0 crescimento é compatível com a idade cronológica, porém, é grande a defasagem em todas as áreas de desenvolvimento.

A aplicação da Escala de Desenvolvimento de Heloisa Marinho evidenciou que algumas apresentam raciocínio lógico e possibilidade limitada de desenvolvimento cognitivo. 0 prognóstico dessas crianças é sombrio em função do grau de comprometimento neurológico, psicológico e social.

0 estudo evidenciou que, para a socialização destas crianças, faz-se necessária grande estimulação e inserção em atividades com pessoas da mesma idade cronológica para servirem de estímulo.

Para efeito positivo no caso da síndrome alcoólica fetal (SAF), além da importância do diagnóstico precoce, seguido das intervenções necessárias, é preciso que todas as pessoas que fazem parte do processo de crescimento e de desenvolvimento destas crianças, sejam elas pais, professores ou cuidadores, se envolvam de forma integral na assistência das suas desabilidades, tendo a consciência de que cada criança é única e demanda diferentes necessidades.

6. Ministério da Saúde (BR). Pacto nacional pela redução da mortalidade materna e neonatal. Brasília (DF); 2004.

7. Marinho H. Escala de desenvolvimento. Rio de Janeiro(RJ): Livro Ltda; 1977.

8. Minayo CS. Pesquisa social: teoria, método e criatividade. Petrópolis(RJ): Vozes; 2001.

9. Lei n 8069 , de 13 de julho de 1990. Dispõe sobre o Estatuto da Criança e do Adolescente e dá outras providências. Diário Oficial da República Federativa do Brasil, Brasília (DF), 16 jul 1990.

10. Sreissguth AP. Recent advances in fetal alcohol syndrome and alcohol use in pregnancy. alcohol in healh and disease. New York (NY): Decker; 2001.

11. Horta WA. Processo de enfermagem. São Paulo (SP): EPU;1979. 